LATEINAMERIKANISCHE

LITERATUR GESCHICHTE 


\section{LATEINAMERIKANISCHE LITERATURGESCHICHTE}

unter Mitarbeit von

Walter Bruno Berg, Vittoria Borsò, Hans Hinterhäuser,

Karl Hölz, Dieter Ingenschay, Christopher Laferl,

Klaus Meyer-Minnemann, Horst Nitschack,

Wolfgang Rössig, Roland Spiller,

Harald Wentzlaff-Eggebert und Gerhard Wild

herausgegeben von Michael Rössner

Mit 360 Abbildungen

2., erweiterte Auflage 
Die einzelnen Kapitel bzw. Abschnitte wurden verfaßt von:

S. 1-10: M. Rössner, S. 10-28: W. Rössig, S. 28-54: M. Rössner, S. 54-103: C. Laferl, S. 105-109: M. Rössner, S. 110-116: M. Rössner/ W. Rössig, S. 116-124: M. Rössner, S. 124-129: C. Laferl, S. 130-137: M. Rössner, S. 137-149: K. Hölz, S. 149-152: G. Wild, S. 152-160: D. Ingenschay, S. 160-166: G. Wild, S. 167-176: W. B. Berg, S. 176-190: H. Hinterhäuser, S. 190-199: G. Wild, S. 200-205: K. MeyerMinnemann/V. Borsò/M. Rössner, S. 205-225: K. Meyer-Minnemann, S. 225-236: M. Rössner, S. 236-254: H. Wentzlaff-Eggebert, S. 255-263: M. Rössner, S. 263-283: V. Borsò, S. 284-294: G. Wild, S. 294-308: D. Ingenschay, S. 309-329: V. Borsò/G. Wild, S. 329-347: W. B. Berg, S. 347-372: M. Rössner, S. 372-393: G. Wild, S. 394-405: M. Rössner, S. 406-423: V. Borsò, S. 423-433: G. Wild, S. 433-443: D. Ingenschay, S. 443-454: V. Borsò, S. 454-465: W. B. Berg, S. 466-481: R. Spiller, S. 482-498: H. Nitschack, S. 499-522: M. Rössner.

Die einleitenden Abschnitte zu den Kapiteln wurden unter Verwendung von Material der Autoren der jeweiligen Kapitel vom Herausgeber gestaltet.

Die Deutsche Bibliothek - CIP-Einheitsaufnahme

Lateinamerikanische Literaturgeschichte / unter Mitarb. von Walter Bruno Berg ... hrsg. von Michael Rössner.

- 2., erw. Aufl. - Stuttgart ; Weimar : Metzler, 2002

ISBN 978-3-476-01858-8

ISBN 978-3-476-01858-8

ISBN 978-3-476-02808-2 (eBook)

DOI $10.1007 / 978-3-476-02808-2$

Dieses Werk einschließlich aller seiner Teile ist urheberrechtlich geschützt. Jede Verwertung außerhalb der engen Grenzen des Urheberrechtsgesetzes ist ohne Zustimmung des Verlages unzulässig und strafbar. Das gilt insbesondere für Vervielfältigungen, Übersetzungen, Mikroverfilmungen und die Einspeicherung und Verarbeitung in elektronischen Systemen.

(C) 2002 Springer-Verlag GmbH Deutschland

Ursprünglich erschienen bei J. B. Metzlersche Verlagsbuchhandlung und Carl Ernst Poeschel Verlag GmbH in Stuttgart 2002

www.metzlerverlag.de info@metzlerverlag.de 


\section{INHALTSVERZEICHNIS}

VORWORT VII

Zur zweiten Auflage XII

INDIGENE LITERATUREN UND DIE FRÜHE KOLONIALZEIT

(1492-1650)

Die Reisen des Kolumbus und die Folgen 1

Azteken und Maya / Das Vizekönigreich Neu-Spanien/Mexiko 10

Das Inka-Reich / Das Vizekönigreich Neu-Kastilien/Peru 28

Informations- und Missionsliteratur aus Brasilien 54

DIE BLÜTE DER KOLONIALLITERATUR (1640-1750)

Urbanes Leben und barocke Literatur 61

"Öffentliche « und "private « Literatur im Vizekönigreich

Neu-Spanien 70

Kulteranismus und Neoklassizismus im Vizekönigreich Peru 83

Brasilianischer Barock und jesuitische Tradition 93

\section{DAS ENDE DER KOLONIALZEIT UND DIE UNABHÄNGIGKEITS- EPOCHE (1750-1830)}

Reformen, katholische Aufklärung, Unabhängigkeitskriege 105

Die Literatur Neu-Spaniens bis zur Unabhängigkeit Mexikos 110

Die Vizekönigreiche Peru, Neu-Granada und Río de la Plata 116

Brasilien: arkadische Dichtung und Unabhängigkeit 124

\section{DIE LITERATUREN LATEINAMERIKAS BIS ZUM MODERNISMO}

(1820-1900)

Das Werden der lateinamerikanischen Staaten und ihrer Identitäten 130 Mexiko im 19. Jahrhundert 137

Die verzögerte Ausbildung von »nationalen Identitäten « und Literaturen in Mittelamerika 149

Die spanischen Kolonien in der Karibik: Unabhängigkeitsideen und Sklaventhematik 152

Die Literaturen Kolumbiens und Venezuelas 160

Die Andenländer im 19. Jahrhundert 167

Cono Sur (Chile, La-Plata-Staaten, Paraguay): Aufbruch zu neuen

Ufern 176

Brasilien bis zum Ende des Kaiserreichs 190 


\section{DER MODERNISMO UND DIE FRÜHEN AVANTGARDE-} BEWEGUNGEN IN LATEINAMERIKA (1880-1930)

Modernismo und Modernisierung: der geschichtliche Wandel um die Jahrhundertwende 200

Der hispanoamerikanische Modernismo 205

Brasilien: Jahrhundertwende und das "modernistische Jahrzehnt « 225

Die hispanoamerikanischen Avantgardebewegungen: ein Überblick 236

\section{NACH DEM MODERNISMO (1920-1970)}

Emanzipation, Revolution, neues Selbstbewußtsein und Rückfall in die Krise 255

Mexiko 1910-1968: der Mythos der Revolution 263

Mittelamerika 1920-1970: die Ungleichzeitigkeit des Gleichzeitigen 284 Die spanischsprachige Karibik vom Modernismo bis zur kubanischen Revolution 294

Die Literaturen Kolumbiens und Venezuelas 1920-1970: periphere Regionen gegen das Zentrum 309

Die Andenländer 1920-1970: die Erfahrung des "Anderen « 329 Cono Sur (Chile, La-Plata-Staaten, Paraguay): die Belebung durch das "populäre Genre « und die Blüte der phantastischen Literatur 347 Brasilianische Literatur 1920-1970: das neue Interesse für die Regionen 372

\section{DIE NEUESTEN ENTWICKLUNGEN (1960-1995)}

Zwischen Militärdiktaturen, Schuldenkrise und der Hoffnung auf einen neuen Anfang 394

Mexiko 1968-1995: das Trauma von Tlatelolco und die Folgen 406 Mittelamerika: die Veränderungen in Nicaragua und ihre Auswirkungen auf die Region 423

Literatur der spanischsprachigen Karibik 433

Kolumbien und Venezuela: Violencia und Aufbau einer demokratischen Identität 443

Literatur der Andenländer: Brüche und Aufbrüche 454

Cono Sur (Chile, La-Plata-Staaten, Paraguay): Terror und seine Verarbeitung in der Literatur 466

Die brasilianische Literatur seit 1960: Militärdiktatur, Wirtschaftswunder und neue "Öffnung « 482

Die lateinamerikanische Literatur an der Schwelle zum 21. Jahrhundert (1989-2002) 499

\section{AUSBLICK 521}

BIBLIOGRAPHIE 523

\section{REGISTER 531}

\section{BILDQUELLEN 564}




\section{VORWORT}

So gebräuchlich uns der Begriff "Lateinamerika « zwecks Unterscheidung vom "großen Bruder" USA geworden ist, so sehr ihn hierzulande ein wachsendes Leserpublikum auch mit einer bestimmten Art von Literatur verbindet, so wenig selbstverständlich ist es, sieht man genauer hin, eine lateinamerikanische Literaturgeschichte in deutscher Sprache zu konzipieren. Das beginnt schon mit dem Begriff "lateinamerikanisch « und der Frage nach seinem Umfang und Inhalt. Natürlich wissen wir hierzulande längst, daß damit die Länder des Kontinents südlich des Rio Grande gemeint sind, von Mexiko bis Feuerland, und daß man in den meisten von ihnen Spanisch spricht und schreibt. Aber ist es tatsächlich allgemein bekannt, daß in Brasilien die Landessprache nicht Spanisch, sondern Portugiesisch ist, und daß der sich gegen Ende des 19. Jahrhunderts herausbildende Begriff "Latein-Amerika " unter anderem dem Wunsch entsprungen ist, sich nicht nur gegen das "angelsächsische " Amerika des Nordens abzusetzen, sondern auch von den ehemaligen iberischen Kolonial-Mutterländern zu emanzipieren und stattdessen an das "modernere" Frankreich mit seiner quasi-mythischen Hauptstadt Paris im Geiste einer vagen "Latinität " anzuschließen?

Hält man sich diese historische Wurzel der im Namen Lateinamerikas enthaltenen "Latinität " vor Augen, dann wird auch deutlich, warum es zu rechtfertigen ist, in einer Geschichte der lateinamerikanischen Literatur französischsprachige Kulturen Amerikas wie die der kanadischen Provinz Québec, Guyanas, Haitis oder der französischen Antillen nicht einzubeziehen. So sehr gerade Haiti für eine Selbstdarstellung der lateinamerikanischen Literatur wie Alejo Carpentiers Konzept des "Wunderbar Wirklichen in Amerika " zum Bezugspunkt geworden ist, so wenige Übereinstimmungen gibt es lange Zeit hindurch im Verhältnis der Haitianer und Antillenbewohner zu der (ehemaligen) Kolonialmacht Frankreich einerseits und dem der »Latein «-Amerikaner zu ihrem europäischen »Kulturmodelland " andererseits (für die Québec-Kanadier liegen die Dinge noch einmal anders).

Freilich könnte auch schon die Einbeziehung von Brasilien in einen quasi vereinheitlichten lateinamerikanischen Kulturraum Bedenken hervorrufen. Brasilien teilt zwar die eben angedeutete Perspektive einer Dreiecksbeziehung: iberische Kolonialmetropole - Lateinamerika - Paris, gegen seine Hereinnahme spricht dennoch manches, vor allem die wechselseitige Nichtbeachtung der hispanoamerikanischen und der brasilianischen Literatur, die bis in die 60er Jahre unseres Jahrhunderts reicht. Aber wenngleich die beiden Literaturen lange Zeit hindurch kaum eine direkte Wechselwirkung aufweisen, so sind sie doch in sehr ähnlichen Kontexten entstanden (die spanische und portugiesische Literaturgeschichte sind eng miteinander verzahnt, die Begegnung mit den amerikanischen Realitäten
Latein-Amerika

Die Einbeziehung

Brasiliens 
Gliederung nach Großräumen ist ebenfalls beiden Literaturen gemeinsam), haben auf ähnliche Herausforderungen zu antworten gehabt und sind daher in sehr produktiver Weise miteinander vergleichbar.

Mit der Einbeziehung Brasiliens ist freilich auch ein grundlegendes Problem angesprochen: Schreibt man eine Literaturgeschichte, so schreibt man üblicherweise entweder die Geschichte der "Weltliteratur « oder die einer "Nationalliteratur ", wie das die bisherigen Bände dieser Reihe auch getan haben; eine solche definiert sich entweder durch eine gemeinsame Sprache oder einen gemeinsamen Staat (oder durch beides); die hier behandelte Literatur ist aber in zwei verschiedenen Sprachen und (seit der Unabhängigkeit) in zwanzig verschiedenen Staaten entstanden. Liegt also in der Idee, all diese Literaturen in einer Geschichte zusammenzufassen, nicht eine ziemlich überhebliche, eurozentrisch-kolonialistische Perspektive, sollten wir nicht lieber zwanzig einzelne "Nationalliteraturgeschichten « nebeneinanderstellen, wie das in einigen ähnlichen Versuchen auch geschehen ist?

Nun, so bestechend dieses Argument auf den ersten Blick erscheint: Es übersieht nicht nur das tatsächlich in den Literaturen der lateinamerikanischen Länder ausreichend dokumentierte Gefühl einer kulturellen Gemeinsamkeit, sondern auch historisch dokumentierbare Zusammenhänge, die in den großen Figuren "reisender "Schriftsteller wie Andrés Bello oder Rubén Darío ebenso zum Ausdruck kommen wie in manchen kontinentalen Bewegungen, unter denen an erster Stelle der »Modernismo « der Jahrhundertwende zu nennen ist. Andererseits dürfen diese nachweisbaren Zusammenhänge nicht zum Anlaß für eine vollständige und künstliche Homogenisierung der hier behandelten Literaturen genommen werden, wie sie - in tatsächlich eurozentrischer Perspektive - vor noch nicht allzu langer Zeit im deutschen Verlagswesen vorherrschend war, als uns jeder lateinamerikanische Autor als Mischung aus echtem Indio, tropischem Fabulierer, machistischem Latin lover und Guerillero verkauft wurde. Die lateinamerikanischen Literaturen (ab hier sei der Plural erlaubt) weisen bei allen Gemeinsamkeiten doch auch grundlegend verschiedene Traditionen auf, die in Geschichte, Bevölkerungsstruktur, wirtschaftlichen Gegebenheiten, literarischer Infrastruktur und vielen anderen Einzelheiten begründet sind; freilich fallen diese Unterschiede nicht immer mit den Staatsgrenzen zusammen. Eine Vermittlung dieser kulturellen Vielfalt, wie sie eine Literaturgeschichte zu leisten hat, bedarf daher einer gewissen Differenzierung, aber nicht notwendigerweise der Zersplitterung in die zwanzig "Einzelliteraturen "; wir haben deshalb das Konzept von "Großräumen « gewählt. Für das spanischsprachige Amerika entsprechen in der Kolonialzeit diese Großräume den beiden ursprünglichen Viżekönigreichen Mexiko und Peru. In der Zeit nach der Unabhängigkeit sind es dann sechs: Mexiko, Mittelamerika, die spanischsprachige Karibik, Kolumbien und Venezuela, die Andenländer und der sogenannte "Cono Sur ", bestehend aus Paraguay, Chile und den beiden La-Plata-Staaten Argentinien und Uruguay. Als letzter Abschnitt tritt jeweils Brasilien hinzu. Die einzige Ausnahme von diesem Konzept, das dem Leser mit ausgeprägt regionalen Interessen auch eine rasche Orientierung ermöglicht, bildet die erwähnte kontinentübergreifende Strömung der Jahrhundertwende, der Modernismo, der zusammen mit den Avantgardebewegungen synthetisch für ganz Hispanoamerika bzw. Brasilien behandelt wird.

Damit sind wir bei der zweiten Problematik einer lateinamerikanischen Literaturgeschichte angelangt, und die liegt eben in der Frage nach der 
"Geschichte«. Zwar hat es immer wieder Versuche gegeben, lateinamerikanische Literatur darzustellen, aber die meisten im deutschen Sprachraum unternommenen beschränken sich auf das 20. Jahrhundert (allenfalls auf das 19. und 20.), wenn sie nicht überhaupt anstelle der $\mathrm{Ge}$ schichte lieber ein Panorama der Literatur seit 1950 zeichnen, eben jener Autoren und Werke, die hierzulande im Gefolge des sogenannten "Booms" besondere Bekanntheit und Beliebtheit erreicht haben. Auch wenn diese Jahre tatsächlich einen gewissen Höhepunkt in der Entwicklung der lateinamerikanischen Literatur darstellen, ist das wiederum eine eurozentrische Perspektive, die sogar um einiges bedenklicher erscheint als die geographische »Homogenisierung ". Es ist nicht nur die in den letzten Jahren zu beobachtende Mode des historischen Romans in Lateinamerika, die uns dazu veranlaßt hat, eine solche Optik zu überdenken und in unserer Darstellung den Lateinamerikanern sozusagen »ihre Geschichte zurückzugeben ", wie das vor 25 Jahren schon die erste und bisher einzige vollständige Literaturgeschichte des Kontinents von Rudolf Grossmann versucht hat; es ist auch, ja vor allem, die Erkenntnis, daß ein Verstehen dieser schönen und reichen Literatur gar nicht möglich ist, ohne ihre frühesten Wurzeln, ohne die am Beginn stehenden traumatischen Erfahrungen des "Kulturschocks", der "Begegnung mit dem Anderen", zu kennen.

Dieser Erkenntnis Rechnung tragend, haben wir frühen Epochen insbesondere der Zeit von der Fahrt des Kolumbus bis zur stabilen Eingliederung in den spanischen bzw. portugiesischen Kultur- und Verwaltungsraum in der Mitte des 17. Jahrhunderts - mehr Raum eingeräumt, als es selbst in von Lateinamerikanern geschriebenen Literaturgeschichten üblich ist. Natürlich ist die Literatur in der Kolonialzeit auch Bestandteil der spanischen bzw. portugiesischen Literatur. Und dennoch: Die Emanzipation von den europäischen Themen beginnt schon mit den Texten des Kolumbus. Die Krise des Selbstverständnisses angesichts der Erfahrung des Anderen, das Erlebnis der Ohnmacht angesichts der Gewalt der Natur sind zwei von vielen dominanten Themen, die immer schon den in diesem Kontinent geschriebenen Texten einen anderen Hintergrund verliehen. Um eine Geschichte des Argentiniers Jorge Luis Borges abzuwandeln: Hätte ein Peruaner in der andinen, noch von indigenen Dorfgemeinschaften geprägten Landschaft den Don Quijote Wort für Wort genauso geschrieben, wie es Cervantes in Europa tat, wäre es dennoch nicht dasselbe Buch; der Petrarkismus der "Antarktischen Akademie « von Lima um 1600, der barocke Gongorismus der mexikanischen, peruanischen und brasilianischen Autoren ist nicht dasselbe wie der Petrarkismus oder Gongorismus ihrer europäischen Kollegen, die ähnliche Texte in Florenz, Salamanca oder Coimbra schreiben; er ist ein absurder, fast magischrealistischer Akt, eine Negation der Umgebung des Schreibenden in einem ästhetischen credo quia absurdum.

Man mußte also nicht auf den so intensiv vermarkteten "Magischen Realismus« eines Asturias, García Márquez oder einer Isabel Allende warten, um von einer spezifisch lateinamerikanischen Schreibweise sprechen zu können. Deshalb darf man diese Autoren unseres Jahrhunderts auch nicht als "sympathische Naturkinder " mißverstehen, sondern sollte sie vor dem Hintergrund der fünfhundertjährigen Entwicklung dieser Schreibweise lesen. Aus durchaus parallelen Erwägungen ist hier übrigens auch die neueste, nach dem "Boom " angesiedelte Entwicklung ausführlicher dargestellt; denn das »Bekannte«, sprich: die großen Autoren und
Vom »Panorama" zur Geschichte der lateinamerikanischen Literaturen

Die Andersartigkeit des Gleichen in Lateinamerika 
Periodisierung nach lateinamerikanischen Kriterien
Werke der Zeit zwischen 1949 und 1975, wird erst dadurch richtig erfaßbar und verstehbar, daß es in den ihm eigentümlichen Kontext der Tradition und des Fortwirkens gestellt und nicht nur mit europäischen Augen betrachtet wird, so wesentlich Wechselwirkungen mit Europa auch in der Geschichte dieser Literatur stets gewesen sind.

Dieser scheinbar paradoxe Versuch, als Europäer auch nicht-europäische Kategorien einzubeziehen und sie zugleich europäischen Lesern zu vermitteln, prägt die Konzeption des vorliegenden Bandes. Er drückt sich auch in der hier verwendeten Periodisierung aus. Es erschien uns nicht sinnvoll - wie es Grossmann 1969 tat -, den eigenständigen Charakter der lateinamerikanischen Literatur dadurch vorzuführen, daß man dennoch ausschließlich europäische Periodenbegriffe auf sie anwendet und dann die Abweichungen in "Amero-Romantik ", "Amero-Realismus ", "AmeroExpressionismus « und "Amero-Existentialismus « dokumentiert. Wir haben stattdessen versucht, uns bei den Perioden nach dem Kontinent eigenen historischen Zäsuren zu orientieren: die "Conquista « mit ihren psychologischen und literarischen Nachwirkungen; die barocke Hofkultur; die katholische Aufklärung und die Unabhängigkeitskriege; die mexikanische, später die kubanische Revolution, schließlich das Scheitern der Militärregimes in den letzten zehn Jahren. Und wir haben auch versucht, die solchen Periodisierungen innewohnende Willkürlichkeit ein wenig dadurch zu mildern, daß die als Grenze festgesetzten Jahreszahlen fast immer "überlappend " gewählt wurden, so daß jedes Kapitel auch die in der vorherigen Periode gelegenen Anfänge der behandelten Strömungen aufnehmen und ihre Nachwirkungen in der folgenden andeuten kann.

Wenn die Literaturen Lateinamerikas sich in diesem Jahrhundert also endgültig von ihren europäischen Mutterländern emanzipiert haben, dann war es hoch an der Zeit, diesen Versuch einer dem lateinamerikanischen Denken und Schreiben möglichst adäquaten Vermittlung in einer deutschsprachigen Literaturgeschichte zu unternehmen, die sich gleichermaßen an ein akademisches Publikum wie an die vielen Freunde lateinamerikanischer Texte unter den Lesern wenden soll. Wir haben versucht, einen Kompromiß zu finden und trotz der prinzipiellen Offenheit für ein nichtakademisches Publikum in den einzelnen Beiträgen auch durchaus ein wissenschaftliches Profil sichtbar werden zu lassen. Dennoch mußte der Herausgeber aus vierzig Einzeltexten von insgesamt dreizehn Autoren, die fünfhundert Jahre Geschichte der literarischen Produktion von zwanzig Ländern behandeln, ein Buch machen; es galt also, erbarmungslos zu kürzen, Übergänge zu finden, die Texte aufeinander abzustimmen, um sie zu einem einzigen, nicht zu sehr nach Collage klingenden Text werden zu lassen. Die Mitarbeiter haben diese "Vergewaltigung " mit bewundernswerter Gelassenheit und Toleranz ertragen; dem Herausgeber bleibt nur, nun auch vom Leser Verständnis für die Unvollkommenheiten des Resultats zu erbitten: Was man Gutes in diesem Buch findet, ist den Autoren zu danken; die - zahlreichen - Schwächen hat der Herausgeber zu verantworten.

Nicht zuletzt bedarf die Geschichte einer immer noch "fremden « Literatur wie der lateinamerikanischen auch sehr oft der Hintergrundinformation, der Aufzählung von manchen Spezialisten banal erscheinenden Fakten. Auch hier galt es, einen Kompromiß zu finden zwischen der zur Vermittlung notwendigen Information und den Möglichkeiten einer auf begrenztem Raum operierenden Literaturgeschichte. Für eine umfassende Einführung in das Phänomen Lateinamerika ist sicherlich die begleitende 
Lektüre eines Geschichtswerkes zu empfehlen. Der erwähnten Orientierung am Leser, der aus Freude an der Literatur liest, wurde durch möglichst umfängliches Bildmaterial und lektüreleitende Randglossen Rechnung getragen, die nicht nur die Literatur selbst, sondern auch die immer noch "fremdartige "Welt Lateinamerikas als Hintergrund der Texte erfahrbar machen sollen. Der Verlag, insbesondere die betreuenden Lektoren und Lektorinnen Petra Wägenbaur, Oliver Schütze, Andrea Rupp und Sybille Paulus sowie Bernd Lutz selbst, hat dabei jede nur mögliche Unterstützung geleistet; immer hilfreich waren auch die Ibero-Amerikanischen Forschungsinstitute in Berlin und Hamburg sowie Thomas Scheerer mit seiner Datenbank BiLA in Augsburg. Ein spezieller Dank gilt schließlich David Lagmanovich und Gustav Siebenmann für ihre Anregungen und meinen Münchner Mitarbeitern Daniela Nardi, Ana Ribeiro-Kügler und Martin Weidlich für ihre unermüdliche Hilfe bei Korrekturlesen, Registererstellung und Komplettierung der Bibliographie.

Die Orientierung dieses Bandes am Leser drückt sich schließlich in dem Bestreben aus, überschaubar zu bleiben: Bei der für jede Literaturgeschichte notwendigen Selektion ist bewußt auf auch nur den Schein enzyklopädischer Vollständigkeit verzichtet worden. Viele Autoren und Werke fehlen; dafür haben wir versucht, statt einer "kommentierten Liste " von Namen und Titeln dem Leser durch die ausführlichere Vorstellung von Autoren und Werken paradigmatischen Charakters ein anschauliches Bild der literarischen Epoche des jeweiligen Raumes mit Ansätzen zu kritischer Betrachtung aus möglichst vielfältiger Perspektive zu liefern. Aus dem Vorstehenden dürfte eines klar geworden sein: Die große Herausforderung bestand darin, einerseits diese Literaturen dem europäischen Leser näher zu bringen, andererseits der Versuchung zu widerstehen, sie ausschließlich mit unseren Kategorien, in einer überheblichbelehrenden eurozentrischen Perspektive zu betrachten. Glücklicherweise gibt es dafür einige Vorbilder. So berichtet einer der frühesten deutschen Hispanoamerikanisten, der Münchner Romanist Karl Vossler, über eine vor 60 Jahren absolvierte Vortragsreise nach Argentinien: »Ich, der ich nach Südamerika gekommen war, um einige Vorträge über Themen meines Faches zu halten, bemerkte bald, daß meine vornehme Mission noch einen anderen Aspekt in sich trug, einen bescheideneren zwar, aber auch einen wichtigeren: Zuhören, Annehmen, mit freundlichem Echo Anregungen geben. "Dieses kurze Programm war es auch, von dem die Autoren der vorliegenden Literaturgeschichte sich leiten ließen. Lateinamerika hat eine große Zahl von Gesichtern; wir haben versucht, möglichst viele von ihnen für möglichst viele deutschsprachige Leser erfahrbar zu machen.

München, im Juli 1995

Michael Rössner 


\section{Zur zweiten Auflage}

Das ungebrochen große Interesse der deutschen Leser an der lateinamerikanischen Literatur hat es notwendig gemacht, nach wenigen Jahren eine zweite Auflage unserer Literaturgeschichte vorzulegen. Dabei ist die Erstausgabe von 1995 durch ein Kapitel über das letzte Jahrzehnt ergänzt worden, gekennzeichnet durch die Eckdaten 1989 (Fall der Berliner Mauer und Zusammenbruch des "real existierenden Sozialismus «) und 2001, als das "globalisierte Weltsystem " durch das Attentat auf das New Yorker World Trade Center erstmals grundlegend in Frage gestellt wurde.

Eine solche Ergänzung konnte schon wegen des relativ geringen Textumfangs nicht in der nach sieben Großräumen gegliederten Form erfolgen; ich denke aber, daß gerade das neue Generationsbewußtsein der Autoren, die in diesem Jahrzehnt zu führenden Repräsentanten der lateinamerikanischen Literatur geworden sind, wie schon zu Zeiten von Modernismo und Avantgarde eine kontinentübergreifende Betrachtung durchaus rechtfertigt. Die Welt der lateinamerikanischen Kultur - und damit auch die der Literatur - war in diesen Jahren gekennzeichnet von einer unerhört starken Präsenz US-amerikanischer Zivilisation in allen Lebensbereichen, aber zugleich auch von einer immer stärkeren Präsenz der "Latin Culture « in den USA selbst. Diese Hybridisierung, das neue Selbstbewußtsein der Lateinamerikaner und die neuen Ausdrucksformen, vor allem im Bereich des Films, sind vorrangig Gegenstand der Ergänzungen dieser Auflage. Der Spaziergang durch die Literatur des letzten Jahrzehnts ist subjektiv (und der Autor/Herausgeber bekennt sich dazu), er will trotz der unübersehbaren Fülle der Texte einen Rest von Erzählcharakter und Anschaulichkeit in der Darstellung erhalten - was impliziert, daß viele, auch wesentliche Texte ungenannt bleiben müssen. Aber eine Literaturgeschichte, die die Gegenwart mitumfaßt, ist immer ein unabgeschlossenes Unternehmen, und die dritte Auflage in einigen Jahren wird hoffentlich die ärgsten Lücken schließen - allerdings wohl nur, um dafür wieder neue aufzureißen.

Es galt, die enorme Vitalität der neuen und neuesten lateinamerikanischen Literatur darzustellen, die sich programmatisch von den alten Klischees des Magischen Realismus und des Macondismo löst und als eine hybride "Bastardliteratur " selbstbewußt zu positionieren sucht - mittlerweile durchaus mit beachtlichem internationalen Erfolg, so daß sogar schon von einem "neuen Boom " gesprochen wurde. So weit sind wir wohl noch nicht, aber diese neue Auflage unserer Literaturgeschichte kann dem deutschsprachigen Leser gegenüber der Erstauflage nicht nur eine Ergänzung, sondern durchaus ein verändertes, faszinierendes Bild dieser reichen und vielgestaltigen Literatur bieten.

München, im Juni 2002

Michael Rössner 